\title{
Intervening response events between identification targets do not always turn repetition benefits into repetition costs
}

\author{
Matthew D. Hilchey ${ }^{1} \cdot$ Jason Rajsic $^{1} \cdot$ Greg Huffman $^{1} \cdot$ Jay Pratt $^{1}$
}

Published online: 6 January 2017

(C) The Psychonomic Society, Inc. 2017

\begin{abstract}
When there is a relatively long interval between two successive stimuli that must be detected or localized, there are robust processing costs when the stimuli appear at the same location. However, when two successive visual stimuli that must be identified appear at the same location, there are robust same location costs only when the two stimuli differ in their responses; otherwise same location benefits are observed. Two separate frameworks that inhibited attentional orienting and episodic integration, respectively, have been proposed to account for these patterns. Recent findings hint at a possible reconciliation between these frameworks-requiring a response to an event in between two successive visual stimuli may unmask same stimulus and same location costs that are otherwise obscured by episodic integration benefits in identification tasks. We tested this hybrid account by integrating an intervening response event with an identification task that would otherwise generate the boundary between same location benefits and costs. Our results showed that the intervening event did not alter the boundary between location repetition benefits and costs nor did it reliably or unambiguously reverse the common stimulus-response repetition benefit. The findings delimit the usefulness of an intervening event for disrupting episodic integration, suggesting that effects from intervening response events are tenuous. The divide between attention and feature integration accounts is delineated in the context of methodological and empirical considerations.
\end{abstract}

Matthew D. Hilchey

matthew.hilchey@utoronto.ca

1 Department of Psychology, University of Toronto, 100 St. George St., Toronto, Ontario M5S 3G3, Canada
Keywords Repetition effects · Attention and memory · Inhibition of return

A complex network of attentional and memory processes causes the visuomotor system to be biased toward or away from select sources of sensory input. Such biases often are observed in the reaction time (RT) and accuracy data obtained from visual cueing procedures (Posner, 1980; Posner, Rafal, Choate, \& Vaughan, 1985). In broad strokes, cueing procedures are used to evaluate how features (location, color, shape, response, etc.) associated with an initial stimulus (the "cue"1) bias responding to a later stimulus (the "target"), which may or may not share features with the cue. Importantly, the responses required by cues and targets (e.g., the task) heavily influence the type of bias observed. On the one hand, when simple detection or localization responses are required to targets and/or cues, responses, over time, typically become slower when the target appears at the same compared with a different location than the cue. We will refer to such effects as spatial repetition costs (SRCs). Conversely, when identity discrimination responses are required to cues and targets, SRCs are typically observed over time only when the cue and target require different responses. Otherwise, responses are faster when cues and targets share a location. We will refer to the latter effects as spatial repetition benefits (SRBs). We refer to the response-mediated division between SRCs and SRBs in

\footnotetext{
$\overline{1}$ We use "cue" to refer to the first stimulus in a sequence of two. The "cue" may or may not require a response and, regardless of whether a cue response is required, the cue may or may not share a response feature with the target. We specify the response requirements to the cue and its relationship to the target as necessary throughout this report. Ultimately, we are using "cue" because we believe that doing so facilitates joint discussion of episodic integration (event coding) and attentional orienting frameworks. The former former does not acknowledge much difference between responding and not responding to a cue if it is identical to the eventual target, even if it probably should (e.g., Taylor \& Donnelly, 2002).
} 
identification tasks as the SRC-SRB boundary. The present study examined the hypothesis that an intervening response event between a cue and target abolishes or alters the established SRC-SRB boundary in stimulus identification tasks.

Before we begin our investigation into the role played by intervening response events, it is necessary to set the stage for the comparison between SRCs and SRBs. One of the best known examples of SRCs comes from the canonical attentional cueing paradigm (Posner \& Cohen, 1984; Maylor \& Hockey, 1985). When the interval between the cue and target onset exceeds approximately $300 \mathrm{~ms}$, responding is slowest to targets appearing near a prior cue's location, even though there is no correlation between the cue and target locations. This bias, sometimes referred to as inhibition of return (IOR), is long-lasting and particularly robust in tasks that require simple detection or localization responses (Samuel \& Kat, 2003; Taylor \& Klein, 2000). Indeed, this SRC is common even when all cue features, including the response made to the cue, repeat as the target (Rabbitt \& Rogers, 1965; Kirby, 1972, 1976; Maylor \& Hockey, 1985; Soetens et al., 1985;

\section{Notebaert and Soetens (1000 ms RSI)}
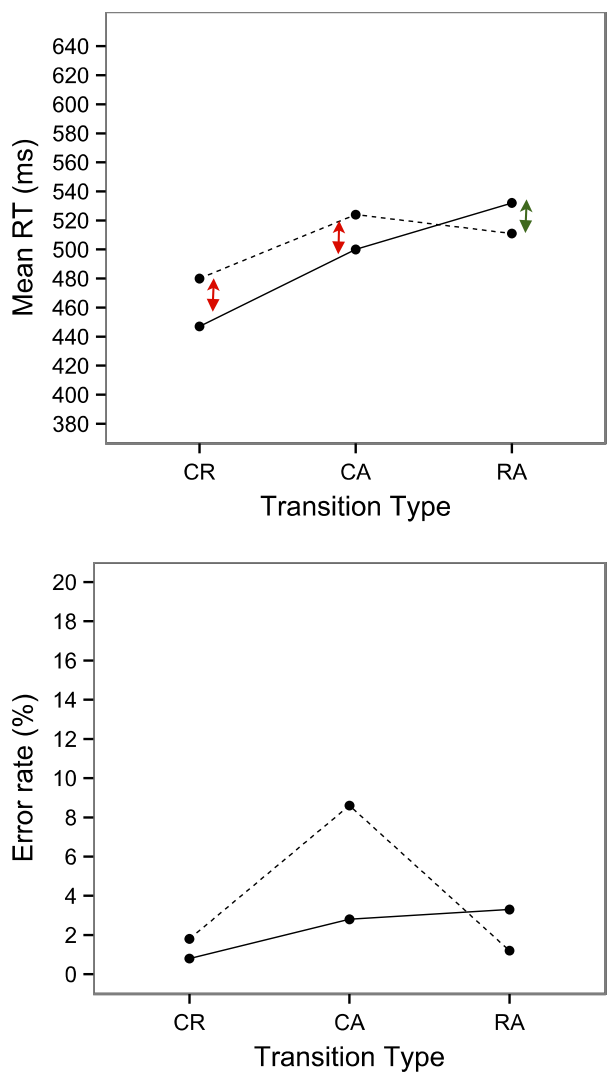

Fig. 1 Typical reaction time (RT) and error rate patterns from Notebaert and Soetens' (2003) cueing procedure. Spatial repetition benefits (SRBs) occur when the cue and target share a response (CR, color repetition; $\mathrm{CA}$, color alternation, response repetition). Spatial repetition costs (SRCs) occur when the cue and target do not share a response (RA, response
Tanaka \& Shimojo, 1996; Soetens, 1998; Taylor \& Donnelly, 2002; Coward, Poliakoff, O’Boyle, \& Lowe, 2004); that goes for detection and localization tasks. In the attention literature, SRCs of this sort are commonly attributed to mechanisms that bias orienting against locations or objects to which attention was involuntarily captured (Klein, 2000).

Whereas SRCs are robust in simple stimulus detection and localization tasks, there is a firmly established boundary on SRCs in stimulus identification tasks at cue-target onset asynchronies (CTOAs) greater than $300 \mathrm{~ms}$ (Terry, Valdes, \& Neill, 1994; Tanaka \& Shimojo, 1996; Pratt \& Castel, 2001; Taylor \& Donnelly, 2002; Taylor \& Ivanoff, 2005). This boundary is a demarcation line, or flashpoint, between inhibited attentional orienting and episodic integration accounts. Most crucially for present purposes, when identity discrimination responses are required to cues and targets, SRCs are observed only when the cues and targets require different responses (Rajsic, Bi, \& Wilson, 2014; Notebaert $\&$ Soetens, 2003). If the cues and targets require the same response, regardless of cue-target identity (e.g., same or different colors), SRBs are observed (Fig. 1). SRBs are thought

\section{Rajsic, Bi and Wilson (1500 ms CTOA)}
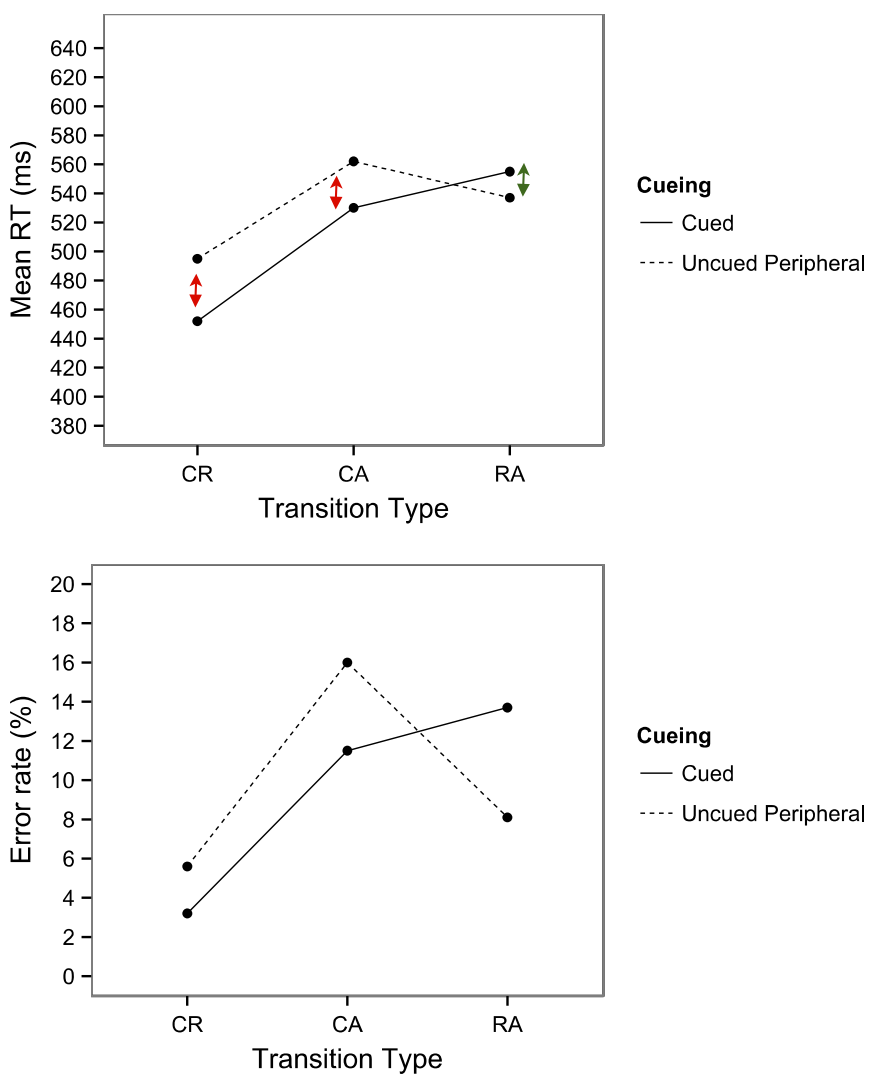

alternation, color alternation). This is the SRC-SRB boundary. Note further that there are processing advantages for stimulus-response repetitions (CR) in either RT, error rate, or both, regardless of the spatial relationship between the cue and target (discussed in paragraph 4 of the introduction) 
to occur because the memory trace of the cue's integrated location-response representation is efficiently re-enacted when the target's location-response representation matches it. In the episodic integration literature, this SRC-SRB boundary can be accounted for by mechanisms that generate costs and/or benefits when the target's representation partially mismatches and perfectly matches/mismatches, respectively, the cue's integrated representation (Hommel, Proctor, \& Vu, 2004; Rajsic et al., 2014; discussed in more detail in the General Discussion). As such, there is rarely a need to appeal to inhibited orienting in identification tasks in which stimuli appear in sequence, because SRCs are conspicuously absent unless there are partial mismatches, on some level, between cue and target events (but see Christie \& Klein, 2001). On the other hand, appeal to episodic integration falls short in detection and localization tasks because SRCs arise even when there is perfect feature (and response) overlap between the cue and target, barring their asynchrony (see Lupiáñez, 2010, for consideration of timing).

Independent of the SRC-SRB boundary, there also are RT benefits when a target matches, compared with mismatches, a previously executed cue response in sequential identification tasks (Bertelsen, 1965; Rabbitt, 1968; Kornblum, 1969). In some cases, this repetition benefit depends on the repetition of the cue stimulus as the target stimulus whereas in other cases response repetition is sufficient (Pashler \& Baylis, 1991; Campbell \& Proctor, 1993; see Tretyak \& Yarrow, 2014, for recent review and extension). In general, stimulus-response repetition benefits in identification tasks are most robust when the interval between the cue response and target onset is $\leq 1 \mathrm{~s}$ (Bertelson \& Renkin, 1966; Hale, 1967), although these repetition benefits can persist unabated well beyond $1 \mathrm{~s}$ when the stimulus-response mappings are many and/or arbitrary (Schvaneveldt \& Chase, 1969). It is a classic stimulus-response repetition benefit that Spadaro, He, and Milliken (2012; also Spadaro \& Milliken, 2013) recently reversed by simply requiring responses to events in between the cue and target. In identification tasks, a reversal of a repetition benefit by a variable other than time in the context of otherwise full feature overlap is remarkably rare; as such, any variable capable of doing so is of significant theoretical interest. The present experimental work was designed to develop a deeper understanding of the nature and robustness of this peculiar event-induced reversal in sequential identification tasks.

The basic conditions for reversing repetition benefits seem simple enough. At fixation, Spadaro et al. randomly presented one of two to-be-discriminated stimuli-each mapping onto a different response and separated by an intervening response event - as a cue and target on a given trial. In each experiment, the two stimuli belonged to a superordinate dimension (e.g., color, line, or word) and stimulus, response or stimulus-response repetition costs (all indistinguishable) were found whenever stimuli were separated by an intervening response event, but not otherwise. Taking color as a presently relevant example (Spadaro et al., 2012, Experiment 1A), a blue rectangle required one manual response and a yellow rectangle another. In one condition, the target appeared 1,500 or $2,500 \mathrm{~ms}$ after the cue response. In this case, responding was faster when the cue repeated as the target. In another condition, an intervening event (e.g., a red circle), which was orthogonal to the identification task, appeared between the cue and target. Whenever a response was made to this intervening event-regardless of its modality (e.g., visual or auditory), response (e.g., pressing both identification response keys simultaneously or verbal), the interval between the cue and target, or whether the intervening event occurred mixed within or separated between blocks - responding was slowest when the cue repeated as the target (Fig. 2). These findings contrasted markedly with Spadaro et al.'s observations of no effect or stimulus-response repetition benefits without intervening response events. To account for the boundary between the stimulus-response repetition benefit and the stimulus-response repetition cost, Spadaro et al. advocated for a two parallel process model. One process, episodic integration, relates to core principles in the aforementioned memory and feature integration literature, in that the retrieval of a response code for a recently encountered stimulus is efficiently reenacted in the absence of updating costs (partial feature mismatches). Critically, Spadaro et al. proposed that responding to an intervening event disrupts this process. The other process is an extension of space-based inhibition of return into the nonspatial domain (Law, Pratt, \& Abrams, 1995; Fox \& de Fockert, 2001; Francis \& Milliken, 2003) with the core idea being that the brain habituates to repeat presentations of the same stimulus (similar to Dukewich \& Boehnke, 2008; Dukewich, 2009). Thus, it is hypothesized that the intervening event knocks out the repetition benefits caused by episodic integration, leaving only repetition costs due to habituation (see also Spadaro \& Milliken, 2013).

The hypothesis that a response to an intervening event is sufficient for knocking out the episodic integration process, of the sort that is also presumed responsible for the SRC-SRB boundary in stimulus identification tasks, has tremendous potential for unifying divergent findings and perspectives derived from simple detection/localization and identification tasks. If, as suggested, a response to a stimulus in between the cue and target reliably disrupts or knocks out the integration process between the cue and the target, it could be very useful for unmasking otherwise overshadowed repetition costs or inhibited orienting effects. In pursuit of this possibility, the present work seeks to evaluate whether Spadaro et al.'s (2012) intervening stimulus-response method (which may be similar to a "cue-back" in the inhibition of return literature; Prime, Visser, \& Ward, 2006) can be used to alter, if not abolish, the SRC-SRB boundary. For this, Notebaert and Soetens' cueing procedure (2003; Experiment 1) is the perfect 


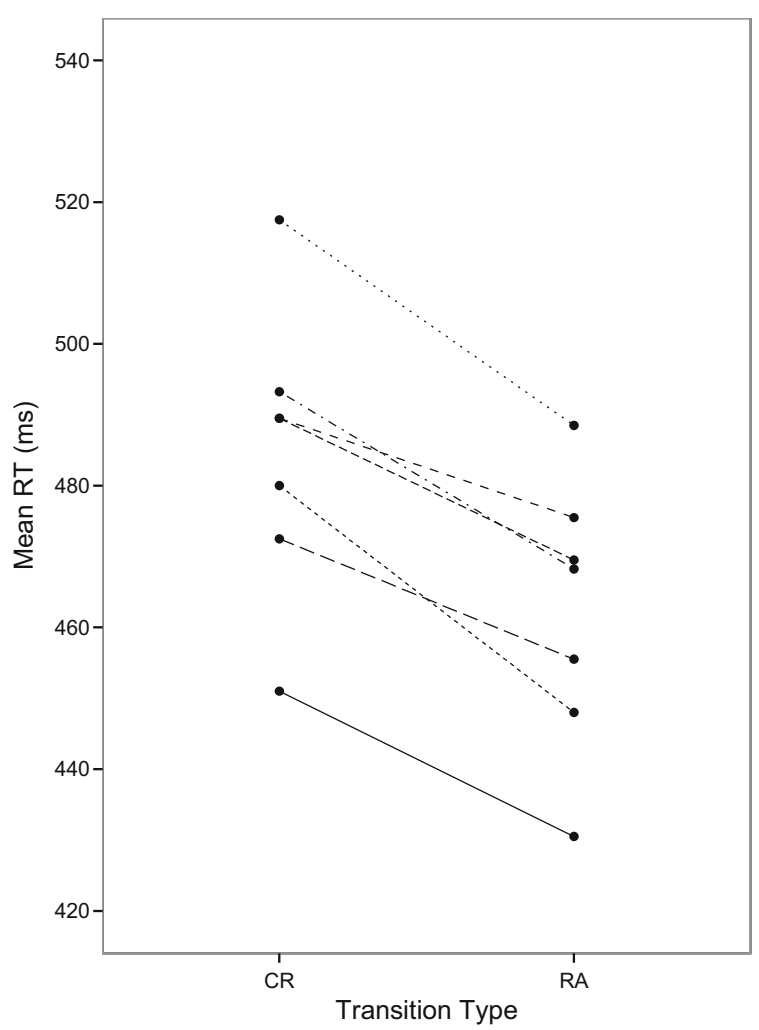

Fig. 2 Typical stimulus-response repetition costs, as observed by Spadaro, He, and Milliken (2012), when a response is required to a stimulus in between the cue response and target response. All cues and targets appeared at fixation. Note that all experiments comprised multiple response stimulus intervals (RSIs), which we collapsed across, because this factor did not matter in any obvious systematic ways. The data from

place to start, in part because its SRC-SRB boundary was recently replicated and extended by Rajsic, $\mathrm{Bi}$, and Wilson (2014) with fixed CTOAs (1,500, 10,500, and 13,500 ms). Thus, there is strong reason to believe that the SRC-SRB boundary is stable in this task in the absence of intervening response events at fixation in between the cue and target. In Experiment 1, we integrated Notebaert and Soetens' (2003) cueing procedure with Spadaro et al.'s procedure primarily to determine whether intervening response events reverse the standard location-response repetition benefit (i.e., unmask spatial inhibition of return). By design, we also have an opportunity to evaluate whether the stimulus-response repetition benefit (presumed same-stimulus habituation or non-spatial inhibition of return) reverses in this more demanding task.

\section{Experiment 1}

In this experiment, the cues and targets randomly took on one of four colors, as in Notebaert and Soetens (2003, Experiment 1), instead of only two, as in Spadaro et al.'s procedure. With this kind of approach, two colors map onto a unique response finger so that the effect of color repetition can be distinguished
Experiment 3B (Color, 50\% intervening visual event trials requiring vocal response; many intermixed RSIs), which likewise showed a stimulus-response repetition cost $(30 \mathrm{~ms})$ is not included because the $\mathrm{y}$ axis would have to be expanded considerably. CR, color repetition (response repetition); RA, response alternation (color alternation)

from the effect of response repetition (Bertelsen, 1965), which is not possible using Spadaro et al.'s two color identification tasks. As in Notebaert and Soetens', the cue and target appeared randomly to the left or right of a central fixation stimulus, but as in Spadaro et al., our cues and targets also appeared randomly at fixation. We used response-stimulus intervals (RSIs) instead of fixed CTOAs, more closely approximating Spadaro et al.'s procedure. Comparisons between Notebaert and Soetens' (2003) and Rajsic et al.'s (2014) findings suggest that similar patterns are obtained (e.g., the SRC-SRB boundary is present) regardless of whether the cue-target interval is fixed or contingent on the response to the cue (Fig. 1), so we have little reason to be concerned that the SRC-SRB boundary hinges on this design choice. Thus in Experiment 1, the cue and target identities, locations, and responses randomly matched or mismatched, allowing for analyses of matching costs and/or benefits.

Spadaro et al.'s intervening response event was integrated into this design as follows: The response to the cue triggered a response-stimulus interval (RSI, $500 \mathrm{~ms}$ ) followed by a centrally-presented intervening response event, the response to which triggered another $500 \mathrm{~ms}$ RSI. Conceptually, this approach yields all cue-target relationships in Spadaro et al. 
and Notebaert and Soetens experiments, whilst ensuring an intervening response event. We also add two new conditions (i.e., a peripheral cue followed by a central target and a central cue followed by a peripheral target), mainly to maintain a balanced design, as appropriately suggested by others (Christie \& Klein, 2001). We ask simply: Will the response to the intervening event, in this context, disrupt or alter the integration process, thereby revealing non-spatial and/or spatial repetition costs instead of benefits? An affirmative to this question would help reconcile inhibited orienting and episodic integration accounts. A negative would delimit Spadaro et al.'s (2012) intervening stimulus-response method for unmasking repetition costs while giving rise to important methodological and theoretical considerations.

\section{Methods}

\section{Participants}

Twenty-four undergraduate students (mean age $=18.9$ years; 18 females) from the University of Toronto consented to participate for course credit. All were naive to the purposes of the experiment and reported normal or corrected-to-normal vision.

\section{Stimuli and apparatus}

All stimuli were displayed on a 17 " CRT monitor connected to a Dell computer running custom Python software. Head position was stabilized by a chin rest $44 \mathrm{~cm}$ from the monitor. Responses were made using the " $z$ " and "/" keys on a standard QWRTY keyboard. All stimuli were displayed on a black background $(0,0,0)$. The three cue and target locationsone left of center $\left(7.5^{\circ}\right)$, one at center, and one right of center $\left(7.5^{\circ}\right)$-were marked by gray (RGB: $\left.128,128,128\right)$ outline ( 1 pixel) placeholder boxes $\left(2^{\circ} \times 2^{\circ}\right)$. The cue and target colors were red (RGB: 128, 0, 0), green (RGB: 0, 128, 0), blue (RGB: 0, 0, 128) and purple (RGB: 128, 0, 128). The cues and targets filled the placeholder boxes. Two different colors mapped onto each index finger; the color-response mappings with completely counterbalanced across participants. Each trial began with a small $\left(0.3^{\circ} \times 0.3^{\circ}\right)$ white (RGB: $255,255,255)$ fixation cross centered in the middle placeholder. The intervening response event was a small white circle (radius $=0.3^{\circ}$ ) in the center placeholder box, to which both keys had to be pressed simultaneously. Text at the end of a trial appeared in the middle of the display in white font.

\section{Procedure}

Each person participated in one block of 432 trials, except one (396 trials). This trial count corresponds with three randomly intermixed runs of all factorial combinations of cue color (red, green, blue, or purple), target color (red, green, blue, or purple), cue location (left, center, or right) and target location (left, center, or right). Each participant sat down with the experimenter before testing and was fully instructed on the task. The experimenter emphasized the stimulus-response mappings, that eye gaze should remain on the center box, and that there was no correlation between the cue and target colors and locations.

The sequence of stimulus events appears in Fig. 3. Each trial began with the fixation cross and placeholder boxes; the cross was extinguished after $500 \mathrm{~ms}$, which signaled the beginning of the trial. The color cue appeared $1 \mathrm{~s}$ later. The response to the cue extinguished the cue and triggered a $500 \mathrm{~ms}$ RSI, after which the intervening (bimanual) response event, as in Spadaro et al., appeared at center. The response to the intervening event extinguished the intervening event and triggered another $500 \mathrm{~ms}$ RSI, after which the color target appeared. If all trial responses were correct, the target response triggered a black screen and a $2 \mathrm{~s}$ intertrial interval (ITI). If a response error occurred at some point during the trial, a feedback screen appeared ("A response error was made..."), which also included all of the response mappings as reminders (e.g., "respond to red and green squares with the ' $z$ ' key..."). Feedback was acknowledged by a response key and triggered a 2 s ITI.

\section{Results}

Central and peripheral targets were analyzed separately. Peripheral target data were analyzed with $3 \times 3$ repeated measures ANOVAs. One factor was Cueing, which refers to the spatial relation between the cue and target [cued, uncued peripheral (cue), or uncued central (cue)]. The other factor, which we call Transition Type (following Notebaert \& Soetens, 2003), represented the relationship between the cue and target's color and response features [(Color Repetition (CR), Color Alternation (CA) with response repetition, or Response Alternation (RA)]. Central target data were analyzed with $2 \times 3$ repeated measures ANOVAs. In this case, Cueing had only two levels [cued, or uncued peripheral (cue)].

\section{Peripheral targets}

Most trials (89.9\%) were completed without error, leaving ample data for RT analysis on error-free trials. Z scores for correct target RTs were computed for each participant at each level (9 levels) of the ANOVA; trials $(3.1 \%)$ containing $\mathrm{z}$ scores greater than 2.5 were excluded as outliers. Next, we examined the data for exceptionally long CTOAs. We considered intervals greater than $5 \mathrm{~s}$ between the cue and target onset to be unreasonably long and thus excluded all such trials $(<1 \%)$. Descriptively, we note that M RTs to the cue and intervening events were $741 \mathrm{~ms}$ and $327 \mathrm{~ms}$, respectively. Accordingly, the mean CTOA was 2,068 ms. 


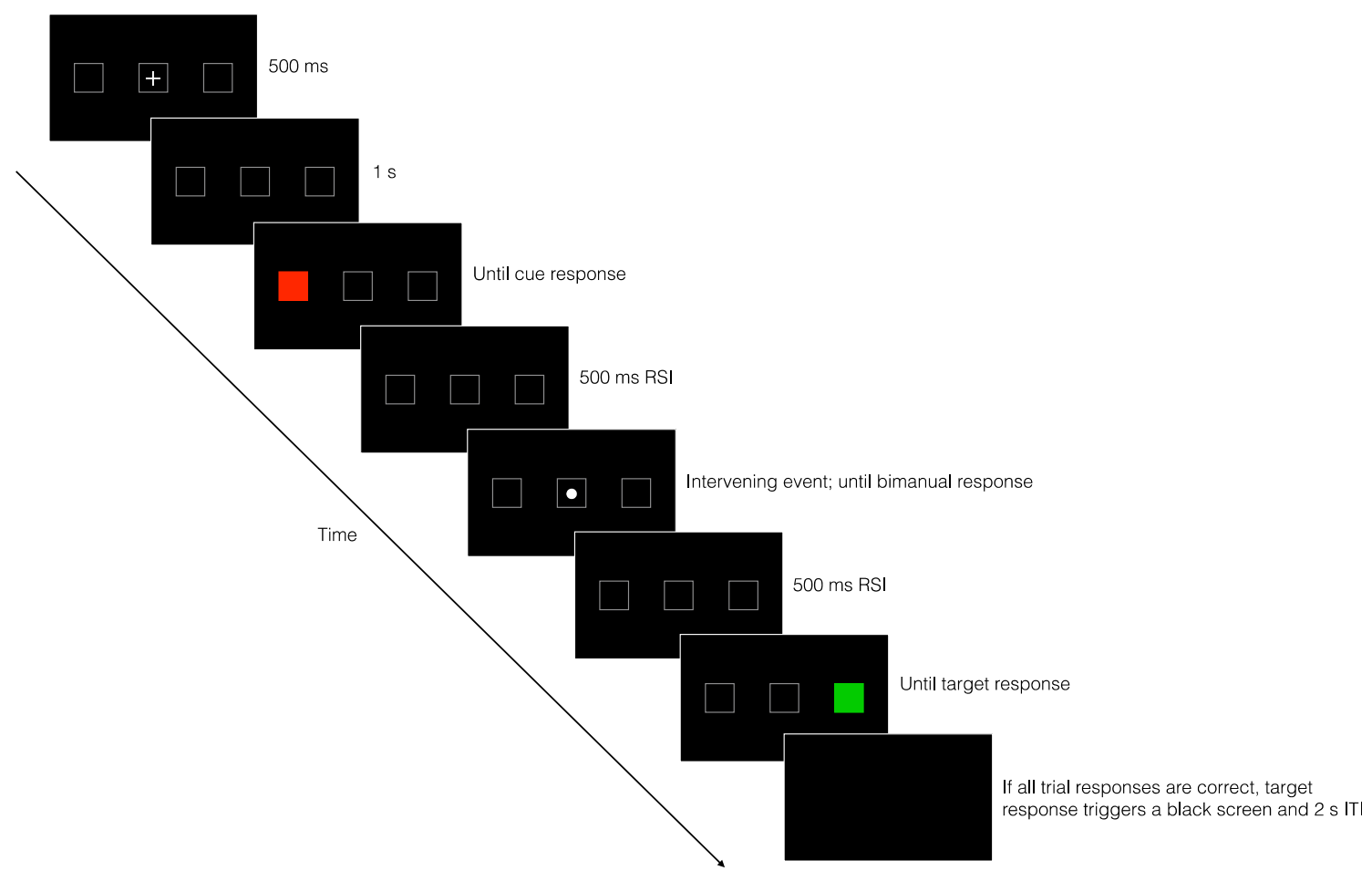

Fig. 3 A sequence of possible stimulus events in this cue-target identification experiment. See "Methods" for more detail

Correct participant M RTs were submitted to a $3 \times 3$ repeated measures ANOVA. The main effects of Cueing $[\mathrm{F}(2,46)=$ $6.36, \mathrm{MSE}=1107, p<0.01]$ and Transition Type $[\mathrm{F}(2,46)=$ 35.53, $\mathrm{MSE}=5410, p<0.01]$ were significant and these effects were qualified by the two-way interaction $[\mathrm{F}(4,92)=$ 10.74, MSE $=1159, p<0.01]$. Replicating Notebaert and Soetens (2003), and Rajsic et al. (2014) (Fig. 4), SRBs were observed when the cue and target shared a response, whereas an SRC was observed when they did not. Also consistent with earlier reports and as can be gleaned from the parallel lines between CR and CA in Fig. 4, the alternating color feature did not interact with Cueing $(\mathrm{F}<1$; i.e., no stimulus-location repetition benefits or costs). The corresponding analysis on error rates to the target on otherwise correct trials revealed similar patterns [Cueing $\mathrm{x}$ Transition Type: $\mathrm{F}(4,92)=9.20$, MSE $=26.74, p<0.01$; Cueing: $F<$ 1; Transition Type: $F(2,46)=31.33, M S E=65.20, p<0.01]$, although the alternating color feature appeared to interact with Cueing when the cue and target shared a response $[F(2,46)=$ 3.25, $M S E=20.0, p<0.05]$; however, this may be because accuracy was near ceiling when color repeated. Regardless, clearly typical patterns were observed and not reversed by the response to the intervening event.

\section{Central targets}

Most trials (90.7\%) were completed without error. As before, zscores were computed and trials containing $\mathrm{Z}$-scores greater than 2.5 were excluded (3.3\%). Trials containing unreasonably long CTOAs ( $>5 \mathrm{~s}$ ) were likewise removed $(<1 \%)$. M RTs to the cue and intervening event were $731 \mathrm{~ms}$ and $327 \mathrm{~ms}$, respectively. Accordingly, the mean CTOA was $2,058 \mathrm{~ms}$.

Correct participant M RTs were submitted to a $2 \times 3$ repeated measures ANOVA (Fig. 5). The main effects of Cueing and the interaction between Cueing and Transition Type were not significant $(\mathrm{Fs}<1)$. There was a main effect of Transition Type $[F(2,46)=31.47$, MSE $=6248, p<0.01]$. Simply, RTs were fastest on CR trials $(481 \mathrm{~ms})$, whereas there was no statistically meaningful difference between CA $(600 \mathrm{~ms})$ and RA (583 ms) trials $[\mathrm{t}(23)=1.15, \mathrm{SE}=14.94, p>0.10]$. Clearly, the intervening response event did not reverse the usual benefit on CR trials (Fig. 5; i.e., there was no evidence for a stimulus-response repetition cost). Although Cueing and Transition Type did not interact in the RT analysis, they did in the error rate analysis $[\mathrm{F}(2,46)=9.66, \mathrm{MSE}=25.74, p<0.01$; also, no main effect of Cueing: $F<1$ but a main effect of Transition Type: $F(2,46)=18.73, M S E=45.20, p<0.05]$. Simply, there was an SRB-SRC boundary in error rate but not, as noted, in RT. Apparently, as documented by Notebaert and Soetens (2003) in a different context (their Experiment 2), alternation biases are more prominent in error rate than RT when a cue differs from a target feature when the target appears at fixation.

\section{Discussion}

Typical SRB and SRC patterns were obtained despite intervening response events. Moreover, comparison between $\mathrm{CR}$ 


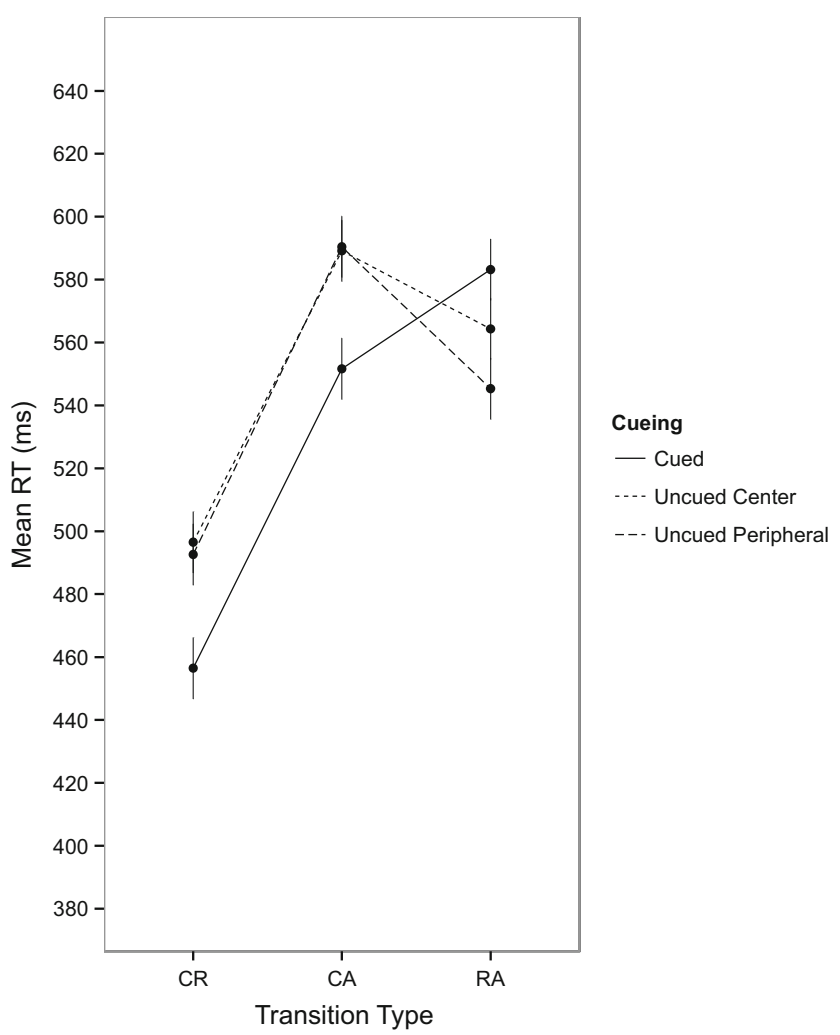

Fig. 4 Left panel: Mean reaction times (RTs) for all combinations of Cueing and Transition Type for peripheral targets in Experiment 1. Right panel: Mean error rate for all combinations of Cueing and Transition Type for peripheral targets in Experiment 1. The error bars

and RA transition types revealed robust stimulus-response repetition benefits, not costs, which occurred regardless of cueing (cf, CR-RA transition types for cued and uncued trials). Although beyond our purview, we note further that these differences imply a level of independence between stimulus-response and location-response representations. In other words, location, stimulus and response features were not completely integrated into a single representation. This is not to say that complete feature integration cannot occur (Hommel et al., 2004) nor does this diminish the hypothetical role of matching and mismatching operations as they apply to latent, binary feature-response representations (Hommel, 2007). These patterns, as they bear on episodic integration and inhibited orienting theories, will be elaborated on in the General Discussion.

\section{Experiment 2}

At this point, it seems that intervening response events do not reverse spatial or nonspatial repetition benefits when more complex coding operations are involved. In broad strokes, we are defining increased complexity by the two major differences between our experiment and those of Spadaro et al.

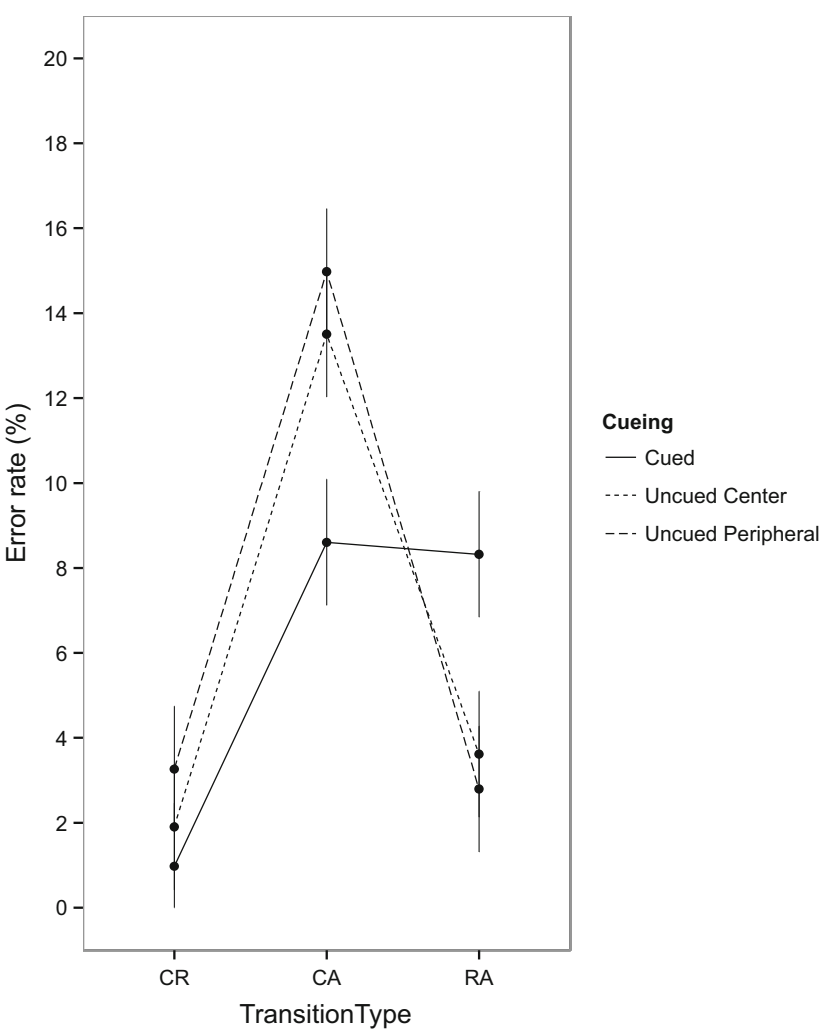

are half Fisher's least significant differences, derived from the mean squared error term of each interaction. CR, color repetition; CA, color alternation (response repetition); RA, response alternation (color alternation)

(2012): four instead of two colors (2:1 response mappings), and three locations instead of a single target location at fixation. One or both of these variables likely mattered. However, because a major theoretical interest in the present report concerns the SRC-SRB boundary, it is not sensible to reduce the number of target locations to one. Thus, for present purposes, we are left contemplating whether our failure to abolish episodic integration processes is due to the increased stimulus-response mappings. That is, paradoxically, maybe our information reduction approach for studying the relative contributions of stimulus and response repetition to the stimulus-response repetition cost fundamentally abolished it. After all, and as noted in the introduction, repetition benefits tend to be persistent and long-lasting in tasks involving arbitrary and/or many-to-one response mappings. Experiment 2 therefore assessed whether the intervening response event reverses repetition benefits in the context of only two stimulus-response mappings (1:1 color-response mappings).

\section{Methods}

\section{Participants}

Twelve young adults (mean age $=21.3$ years; 8 females) consented to participate for monetary compensation or course 


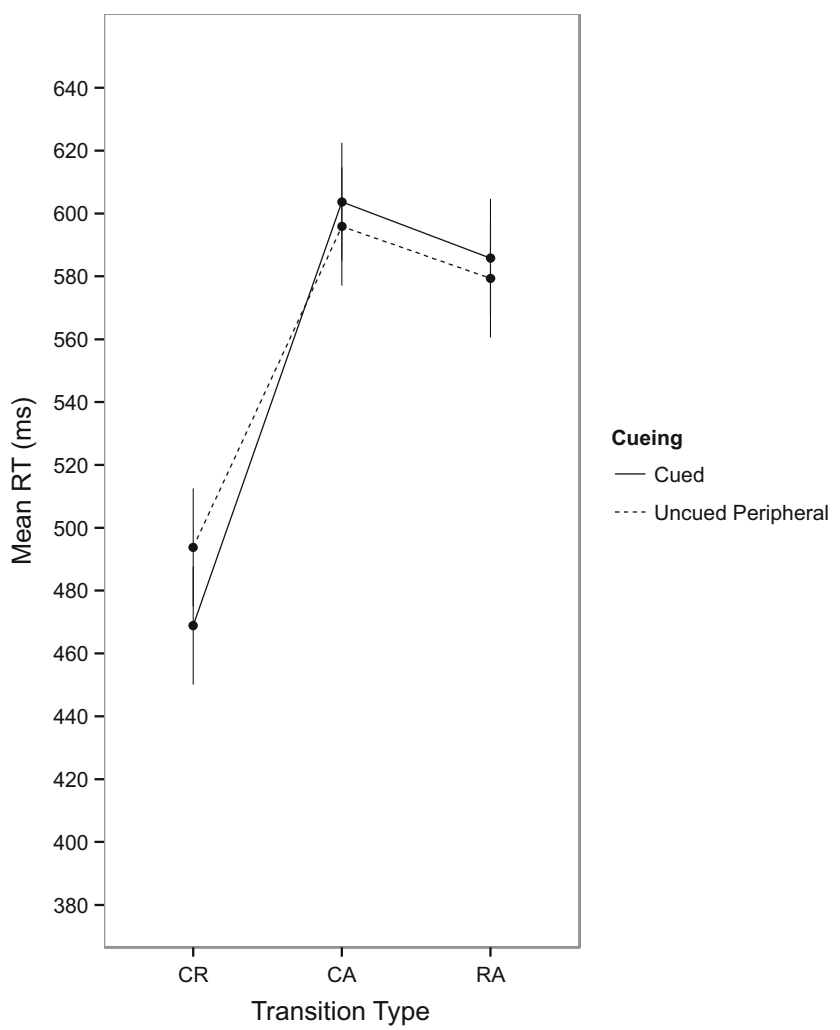

Fig. 5 Left panel: Mean reaction times (RTs) for all combinations of Cueing and Transition Type for central targets in Experiment 1. Right panel: Mean error rate for all combinations of Cueing and Transition Type for central targets in Experiment 1. The error bars are half Fisher's least

credit. None had participated in Experiment 1. All were naive to the purposes of the experiment and reported normal or corrected-to-normal vision.

\section{Stimuli and apparatus}

These were identical to Experiment 1 unless noted. We reduced the number of target colors from 4 to 2 , such that a single color mapped onto each index finger (e.g., red $=\mathrm{z}$ and green $=/$ ). Each participant was assigned a unique set of stimulus-response mappings from the original set of four colors, which allowed us to exhaust all twelve combinations.

\section{Procedure}

This was identical to Experiment 1 except the interval between the response to the cue and the onset of the intervening response event was randomly 500 or $700 \mathrm{~ms}$. The temporal uncertainty was added to replicate the interval between the cue response and intervening event in Spadaro et al. (2012), although we had little theoretical reason for doing so.

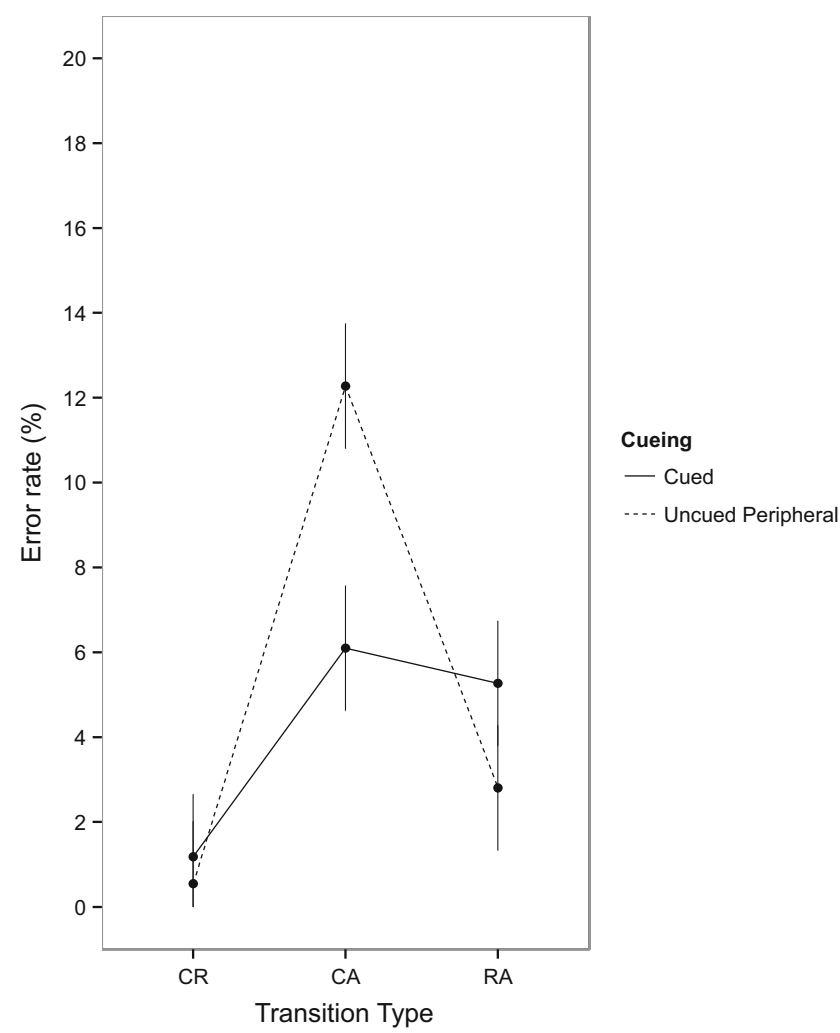

significant differences, derived from the mean squared error term of each interaction. $\mathrm{CR}$, color repetition; $\mathrm{CA}$, color alternation (response repetition), RA, response alternation (color alternation)

\section{Results}

Peripheral and central target data were analyzed with $3 \times 2$ and $2 \times 2$ repeated measures ANOVAs, respectively. Transition Type now comprised 2 (CR and RA) instead of three levels since the change to 1:1 color-response mappings eliminated the CA condition.

\section{Peripheral targets}

Most trials $(88.6 \%)$ were completed without error. Trials (3.0\%) containing z-scores greater than 2.5 were excluded as outliers. Trials $(<1 \%)$ with unreasonably long CTOAs $(>5 \mathrm{~s})$ were also excluded. M RTs to the cue and intervening event were $538 \mathrm{~ms}$ and $339 \mathrm{~ms}$, respectively.

The ANOVA on mean RTs revealed an effect of Transition Type $[F(1,11)=15.35, M S E=765, p<0.05]$, i.e., stimulus-response repetition costs, and a marginal effect of Cueing $[F(2,22)=2.79, M S E=159, p<0.10]$. This main effect of Transition Type, however, was qualified by the two-way interaction $[F(2,22)=25.79, M S E=624, p<$ 0.05], which showed the SRB-SRC boundary (Fig. 6). The corresponding analysis on error rates revealed no main effects (Cueing: $F<1$; Transition Type: $F<1$ ) but an interaction $[F(2,22)=16.13, M S E=23.2, p<0.05]$ that resembled the 


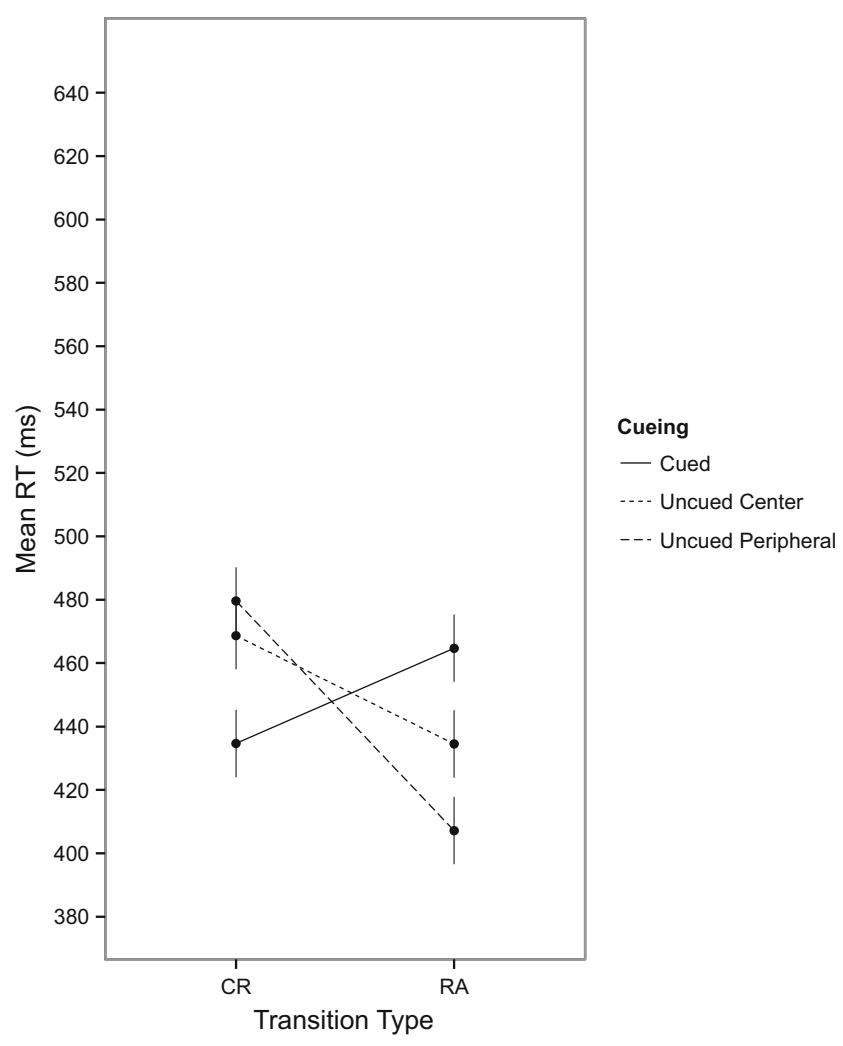

Fig. 6 Left panel: Mean reaction times (RTs) for all combinations of Cueing and Transition Type for peripheral targets in Experiment 2. Right panel: Mean error rate for all combinations of Cueing and Transition Type for peripheral targets in Experiment 2. The error bars

RT patterns. Simply, an SRB was observed when the cue and target shared a response, whereas an SRC was observed when they did not.

\section{Central targets}

Most trials (92.8\%) were completed without error. Trials $(2.8 \%)$ with RT outliers were excluded. Trials $(<1 \%)$ containing unreasonably long CTOAs were excluded. M RTs to the cue and intervening event were $530 \mathrm{~ms}$ and $345 \mathrm{~ms}$, respectively.

RT analyses revealed no main effects [Cueing: $F(1,11)=$ 2.62, $M S E=570, p>0.05$; Transition Type: $F(1,11)=1.80$, $M S E=460, p>0.05]$. There was an interaction $[F(1,11)=$ 9.24, $M S E=217, p<0.05]$. SRBs were robust when the cue and target shared a response; this appears to have occurred because RTs on uncued CR trials were particularly slow relative to all other conditions, which produced roughly equivalent RTs (Fig. 7). The corresponding analysis on error rates revealed a marginal effect of Cueing $[F(1,11)=3.47, M S E=$ $11.44, p<0.10]$, suggesting more errors on cued trials, but no effect of Transition Type or an interaction $(F \mathrm{~s}<1)$. Collectively, although the SRC was surprisingly weak or absent, it is clear that the intervening response event did not

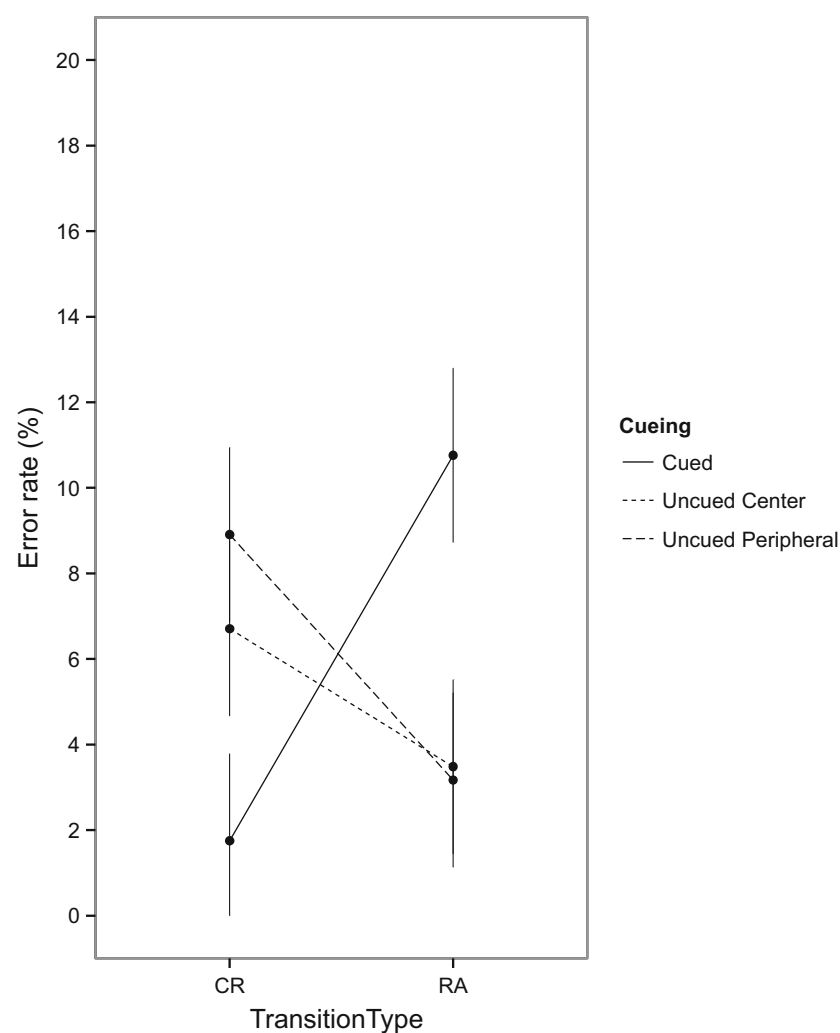

are half Fisher's least significant differences, derived from the mean squared error term of each interaction. CR, color repetition; CA, color alternation (response repetition); RA, response alternation (color alternation)

reverse the SRB, neither is there unambiguous evidence that the stimulus-response repetition benefit was reversed.

\section{Discussion}

The peripheral target findings demonstrated an SRC-SRB boundary that remained intact despite the intervening response event (Terry, Valdes, \& Neill, 1994, Experiment 3; Notebaert, Soetens, \& Melis, 2001, Experiment 2; Taylor \& Donnelly, 2002, Experiment 1; for similar observations without intervening response events). In fact, this boundary was qualitatively very similar to what we observed in Experiment 1 . The central target data, although apparently less sensitive to the SRC-SRB boundary, also revealed patterns consistent with the boundary.

Whereas the SRC-SRB boundary remained intact, the stimulus-response repetition benefit was clearly altered. Comparison between Experiments 1 and 2 on the CR and RA transition types demonstrates that the effect of stimulusresponse repetition was dramatically reduced, and arguably reversed (see next paragraph), when the task involved fewer stimulus-response mappings and an intervening response event. As such, on some level, stimulus-response and location-response repetition effects are dissociable 


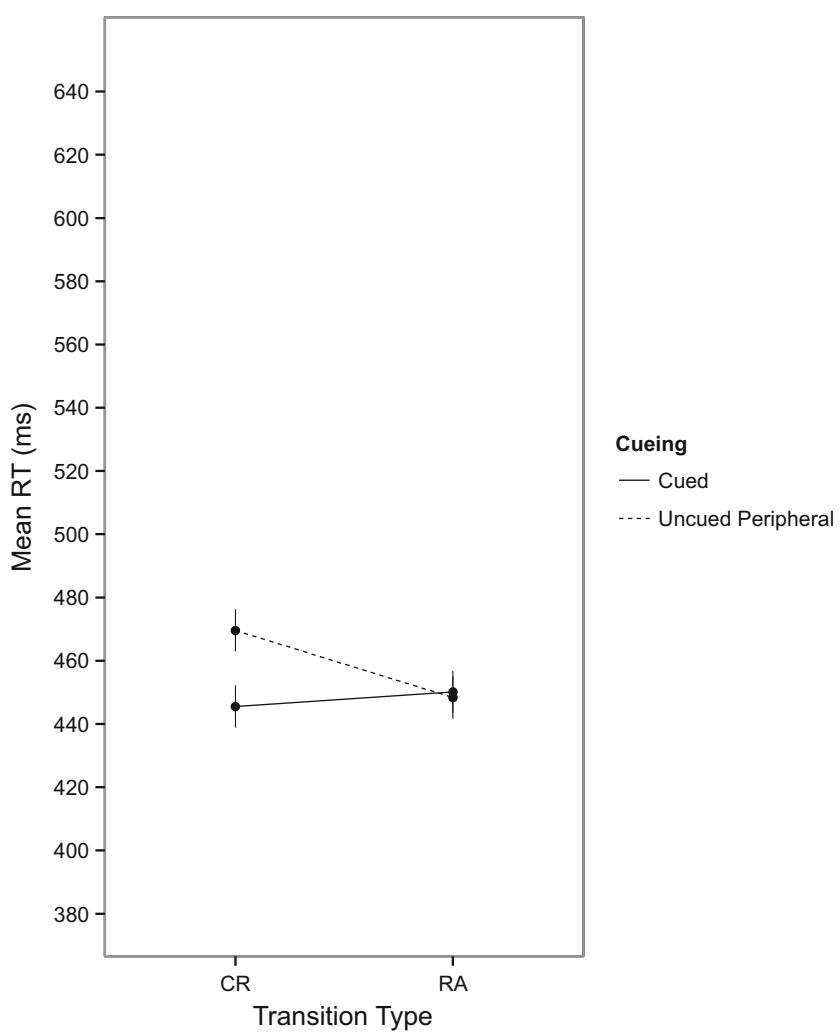

Fig. 7 Left panel: Mean reaction times (RTs) for all combinations of Cueing and Transition Type for peripheral targets in Experiment 2. Right panel: Mean error rate for all combinations of Cueing and Transition Type for peripheral targets in Experiment 2. The error bars

(Notebaert \& Soetens, 2003 and Notebaert, Soetens, \& Melis, 2001). Finally, the comparison between cued CR and RA central target data, which are analogous to the conditions run by Spadaro et al. (2012), demonstrate weak to nonexistent repetition benefits, not costs. Thus, for the time being, the conditions in which stimulus-response repetition costs can be observed definitively for stimuli appearing at the same location remain confined to two alternative forced choice tasks that involve 1:1 stimulus-response mappings and a single target location at fixation (i.e., Spadaro et al.).

Despite this, there are a couple of ways of contemplating the role of stimulus-response repetition in this experiment, one of which presumes a stimulus-response repetition cost. On the one hand, because cued CR trials and uncued RA trials are full location-response matches and mismatches, respectively, whereas uncued CR and cued RA trials are partial location-response mismatches, one might argue that effect of stimulus-response repetition to the overall patterns is pretty negligible; simply, performance is generally worst for location-response partial mismatches. On the other hand, an alternative interpretation might recognize that the intervening response event plausibly reversed the stimulus-response repetition benefit, even though we did not observe poorer performance on cued CR relative to cued RA transition types like Spadaro et al. did. Although we used only two stimulus-response mappings, the arbitrariness of the

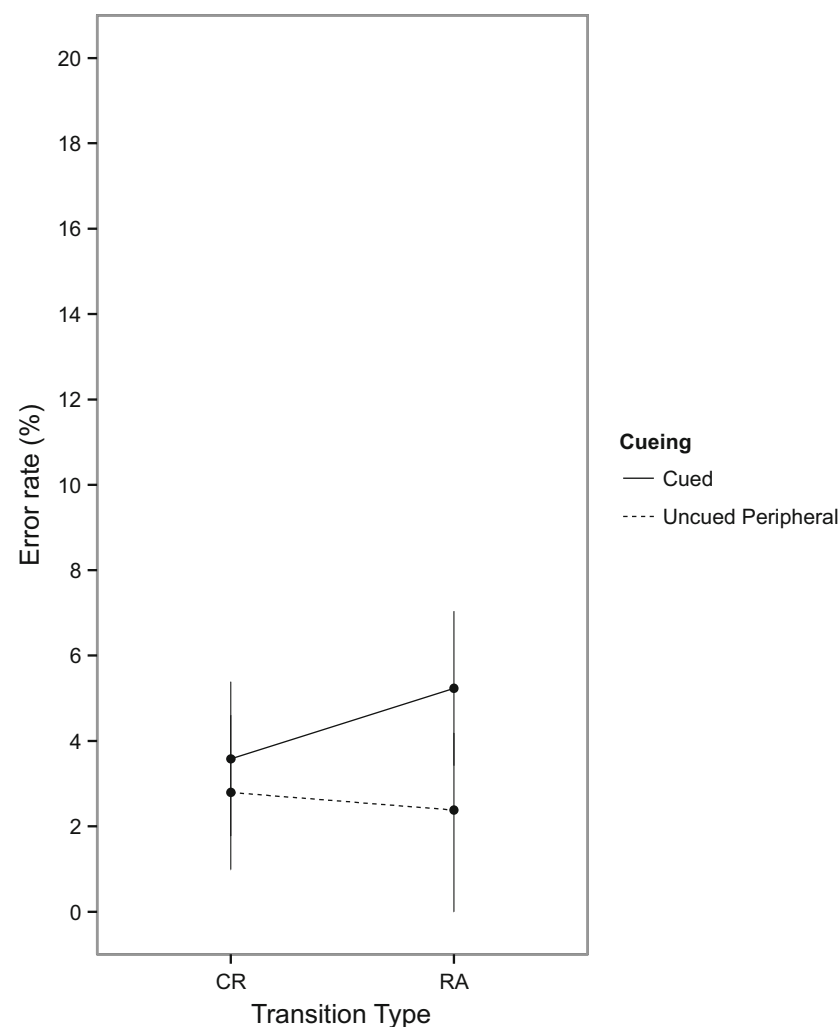

are half Fisher's Least Significant Differences, derived from the mean squared error term of each interaction. CR, color repetition; CA, color alternation (response repetition); RA, response alternation (color alternation)

color-response mapping and our RSI leads us to believe that stimulus-response repetition benefits would have occurred without intervening response events. Indeed, Spadaro et al. (2012) often demonstrated stimulus-response repetition benefits for color targets at CTOAs longer than ours $(>2,500 \mathrm{~ms})$ without intervening response events. We speculate that the intervening response event may have reversed, not just eliminated, the stimulus-response repetition benefit but that this may have been overshadowed by a parallel, opposing and more potent location-response repetition benefit. Implicit to this, is the assumption that persistent location-response repetition benefits occur mainly when the task involves multiple possible target locations (i.e., target location uncertainty); this assumption is needed to account for why Spadaro et al. (2012) were able to observe stimulus-response repetition costs unambiguously across all experiments. If there are hidden stimulus-response repetition costs on some level here but not in Experiment 1, this is a rather inauspicious twist of fate (a catch-22): The mere act of decoupling stimulus from response processes in the stimulus-response repetition cost by using 2:1 stimulus-response mappings seems to strengthen stimulus-response repetition benefits, rendering them irreversible by intervening response events. Regardless, the main point here is that location-response repetition benefits are not abolished or reversed by intervening response events, whereas 
stimulus-response repetition costs emerge unequivocally in very constrained circumstances indeed.

\section{General discussion}

In Experiment 1, we applied Spadaro et al.'s (2012) intervening response event method, which can reverse the common stimulus-response repetition benefit in cue-target identification tasks, to Notebaert and Soetens' (2003) cueing procedure that robustly generates stimulus-response repetition benefits and shows an SRC-SRB boundary in cue-target identification tasks. We did this in an effort to abolish or alter the episodic integration process, which, in theory, masks non-spatial and/or spatial repetition costs in cue-target identification tasks. Contrary to the hypothetical effect of an intervening response event on episodic integration, the present experiment was a near-perfect replication of the findings of Notebaert and Soetens (2003) and Rajsic et al. (2014), which can be seen by juxtaposing Figs. 3 and 1 (peripheral targets). Simply, SRBs were observed when the cues and targets shared a response whereas SRCs were observed when the cues and targets did not share a response. For these peripheral targets, there was likewise no indication that performance was worse when the cue repeated as the target as compared to when it did not; in fact, performance was vastly superior when the cue repeated as the target, regardless of where it appeared. On trials in which the cue and target appeared at fixation, performance was unambiguously best, not worst, when the cue repeated as the target. These findings are simply contrary to those reported by Spadaro, et al.'s (see also Spadaro \& Milliken, 2013) and thus impose a limit on the effectiveness of an intervening response event for reversing stimulusresponse repetition benefits, among other benefits (e.g., SRBs). In fact, our central target findings are qualitatively identical to those in Notebaert and Soetens' (2003) Experiment 2. In that experiment, there was no intervening response event, all colored stimuli appeared at fixation and shape was the irrelevant dimension.

In the critical manipulation in Experiment 2, we reduced the number of target colors from 4 to 2 , such that a single color mapped onto each finger, as in Spadaro et al. (2012). This allowed us to continue investigating the effect of the intervening response event on the established SRC-SRB boundary while simultaneously examining whether the stimulus-response repetition cost would be restored in a two-alternative forced choice task with 1:1 stimulusresponse mappings. From an empirical point of view, we reasoned that reducing the number of stimulus-response mappings could restore the stimulus-response repetition cost from an intervening response event; this is because, as noted in the introduction, stimulus-response repetition benefits are generally more fragile in tasks with fewer stimulus-response alternatives. Empirically, and similar to in Experiment 1, Experiment 2 revealed an
SRC-SRB boundary. However, dissimilar to Experiment 1, Experiment 2 demonstrated non-existent, or plausibly reversed, stimulus-response repetition benefits, even though comparisons between cued CR and RA trials, as in Spadaro et al. (2012), did not reveal a reversal. Despite some ambiguity regarding the stimulus-response repetition cost (or lack thereof), the divergence between experiments, and the observation in Experiment 1 that stimulus-response repetition benefits do not depend critically on location repetition, dissociates an aspect of the stimulus-response from the location-response effects.

How do these findings bear on inhibited attention and episodic integration accounts? Simply, inhibited orienting is not necessary to account for these data (Terry, Valdes, \& Neill, 1994; Notebaert, Soetens, \& Melis, 2001). Regarding inhibition more generally, it is unnecessary to assume inhibition over the cue's response (Druey, 2014; response inhibition), location (Posner, Rafal, Choate, \& Vaughan, 1985; inhibition of return) or identity (Law, Pratt, \& Abrams, 1995; nonspatial inhibition of return) following cue onset, except perhaps in Experiment 2. Generally, it is difficult to devise a framework for the present findings that retains the notions of feature inhibition (spatial, nonspatial, or otherwise), unless a variety of less parsimonious assumptions are made. Instead, the data are reasonably well-accounted for by the matching and mismatching operations espoused by the theory of event coding (TEC; Hommel, Müsseler, Aschersleben, \& Prinz, 2001), which apply to independent feature-response bindings (Hommel, 2007), as proposed by Hommel, Proctor and Vu (2004) for Notebaert and Soetens' (2003) original data.

According to the TEC, since stimulus and response features are coded for by independent and distributed, but interactive feature networks, (Hommel, 2009; Hommel \& Colzato, 2004), it is necessary to integrate stimulus features with their corresponding response features into at least binary featureresponse representations (Hommel, 2007). The integrated representation between a stimulus feature and its response feature is often referred to as an event file. In our experiments, location-response and stimulus- response representations were thus, on some level, independent event files. The TEC proposes that when a new event shares a feature with a prior event, the memory trace of the old event is necessarily retrieved (Hommel, 1998). When there is a perfect match between two events, the prior event response is efficiently reenacted without updating costs. However, when a new event matches an old event on a feature but mismatches on another, there is a conflict between event features; accordingly, these conflicts, which precipitate event-updating costs, disadvantage responding. When all features of a new stimulus event mismatch the prior event, the new event retrieves no existing representation - nothing needs to be updated - and, thus, there are no updating costs. Generally, matching and mismatching operations, when applied separately to the 
location-response and stimulus-response representations and then added together, can account for the data in Experiment 1. Granted, a complete account would seem to require a generic, and additive, bias in favor of the prior response or stimulus. In Experiment 2, matching and mismatching operations can account for the SRC-SRB boundary. Performance tends to be worst when there are partial mismatches between the cue and target location-response events.

Overall, the TEC remains a powerful framework for accounting for the present results. However, the TEC's potential for unifying the cueing literature remains challenged by studies demonstrating repetition costs when cue and target features fully match, findings for which inhibited attention frameworks are commonly invoked. In fact, the class of cueing study that demonstrates repetition costs when all cue and target features overlap, including their responses, poses the most obvious and formidable challenge to episodic retrieval accounts that rely primarily on TEC-like matching and mismatching operations. Simply, retrieval accounts like these have no obvious way of accounting for full cue-target repetition costs via updating (see Tipper, Weaver, \& Milliken, 1995, for similar points on negative priming). Concerning stimulus-response repetition costs, Spadaro et al. (2012) have demonstrated their robustness in simple stimulus-identification tasks with intervening response events. Perhaps more importantly still, recent data suggest that stimulus-response repetition costs may be observed in simple stimulus-identification tasks at fixation without intervening response events, as long as the interval between the cue and target is extended beyond the typical CTOA (Ding, He, Satel, \& Wang, 2016; Avery, Cowper-Smith, \& Westwood, 2015). It thus appears as if, at least in relatively simple two stimulus identification tasks at fixation, some form of inhibition may - eventually - follow from the initial cue representation. However, the present, relatively more demanding experiments, do not convincingly demonstrate stimulus-response repetition costs, presumably because the prior stimulus-response (or stimulus) representation is still active at the time of the target (Experiment 1) or because they are overshadowed by potent location-response repetition benefits (Experiment 2). As far as the SRC side of the SRC-SRB boundary is concerned, there are a number of examples of full repetition costs, even when simple detection responses are required (Maylor \& Hockey, 1985; Coward, Poliakoff, O'Boyle, \& Lowe, 2004). The literature clearly reveals that these full repetition SRCs, which perhaps reflect inhibited spatial orienting, are most likely to be observed in tasks involving limited top-down control and simple coding operations. Yet, as shown, in more complex coding tasks, the latent memory trace of the cue's integrated location-response representation withstands the intervening response event and goes on to generate an SRC-SRB boundary that can be accounted for by episodic integration costs, without any appeal to inhibited attention. At the level of the location-response representation, it appears as though, overall, responding - which may also bias attentional orienting (Smith \& Schenk, 2012) - remains biased toward, not away, from locations that once contained stimuli whose identities mattered for behavior (Maljkovic \& Nakayama, 1996). Thus, at a level that can affect orienting, there is a bias to return to locations that contained actionable search objects and, as shown, this bias is remarkably resistant to intervening response events.

Acknowledgements JP was supported by an NSERC discovery grant. MDH was supported by NSERC and the Killam Trust.

\section{References}

Avery, B., Cowper-Smith, C. D., \& Westwood, D. A. (2015). Spatial interactions between consecutive manual responses. Experimental Brain Research, 233(11), 3283-3290.

Bertelsen, P. (1965). Serial choice reaction-time as a function of response vs. signal-and-response repetition. Nature, 206, 217-218.

Bertelson, P., \& Renkin, A. (1966). Reaction times to new versus repeated signals in a serial task as a function of response-signal time interval. Acta Psychologica, 25, 132-136.

Campbell, K. C., \& Proctor, R. W. (1993). Repetition effects with categorizable stimulus and response sets. Journal of Experimental Psychology: Learning, Memory, and Cognition, 19(6), 1345-1362.

Christie, J., \& Klein, R. M. (2001). Negative priming for spatial location? Canadian Journal of Experimental Psychology/Revue Canadienne de Psychologie Expérimentale, 55(1), 24.

Coward, R. S., Poliakoff, E., O'Boyle, D. J., \& Lowe, C. (2004). The contribution of non-ocular response inhibition to visual inhibition of return. Experimental Brain Research, 155(1), 124-128.

Ding, Y., He, T., Satel, J., \& Wang, Z. (2016). Inhibitory cueing effects following manual and saccadic responses to arrow cues. Attention, Perception, \& Psychophysics, 1-10.

Druey, M. D. (2014). Response-repetition costs in choice-RT tasks: Biased expectancies or response inhibition? Acta Psychologica, $145,21-32$.

Dukewich, K. R. (2009). Reconceptualizing inhibition of return as habituation of the orienting response. Psychonomic Bulletin \& Review, $16(2), 238-251$.

Dukewich, K. R., \& Boehnke, S. E. (2008). Cue repetition increases inhibition of return. Neuroscience Letters, 448(3), 231-235.

Fox, E., \& de Fockert, J. W. (2001). Inhibitory effects of repeating color and shape: Inhibition of return or repetition blindness? Journal of Experimental Psychology: Human Perception and Performance, 27(4), 798-812.

Francis, L., \& Milliken, B. (2003). Inhibition of return for the length of a line? Perception \& Psychophysics, 65(8), 1208-1221.

Hale, D. J. (1967). Sequential effects in a two-choice serial reaction task. The Quarterly Journal of Experimental Psychology, 19(2), 133141.

Hommel, B. (1998). Event files: Evidence for automatic integration of stimulus-response episodes. Visual Cognition, 5(1-2), 183-216.

Hommel, B. (2007). Feature integration across perception and action: Event files affect response choice. Psychological Research, 71(1), $42-63$.

Hommel, B. (2009). Action control according to TEC (theory of event coding). Psychological Research PRPF, 73(4), 512-526.

Hommel, B., \& Colzato, L. (2004). Visual attention and the temporal dynamics of feature integration. Visual Cognition, 11(4), 483-521. 
Hommel, B., Müsseler, J., Aschersleben, G., \& Prinz, W. (2001). Codes and their vicissitudes. Behavioral and Brain Sciences, 24(05), 910 926.

Hommel, B., Proctor, R. W., \& Vu, K. P. L. (2004). A feature-integration account of sequential effects in the Simon task. Psychological Research, 68(1), 1-17.

Kirby, N. H. (1972). Sequential effects of serial reaction time. Journal of Experimental Psychology, 96(1), 32-36.

Kirby, N. H. (1976). Sequential effects in two-choice reaction time: Automatic facilitation or subjective expectancy? Journal of Experimental Psychology: Human Perception and Performance, 2, 567-577.

Klein, R. M. (2000). Inhibition of return. Trends in Cognitive Sciences, 4(4), 138-147.

Kornblum, S. (1969). Sequential determinants of information processing in serial and discrete choice reaction time. Psychological Review, 76(2), 113-131.

Law, M. B., Pratt, J., \& Abrams, R. A. (1995). Color-based inhibition of return. Perception \& Psychophysics, 57(3), 402-408.

Lupiáñez, J. (2010). Inhibition of Return. In A. C. Nobre \& J. T. Coull (Eds.), Attention and time (pp. 17-34). Oxford: Oxford University Press.

Maljkovic, V., \& Nakayama, K. (1996). Priming of pop-out: II. The role of position. Perception \& Psychophysics, 58(7), 977-991.

Maylor, E. A., \& Hockey, R. (1985). Inhibitory component of externally controlled covert orienting in visual space. Journal of Experimental Psychology: Human Perception and Performance, 11(6), 777-787.

Notebaert, W., \& Soetens, E. (2003). The influence of irrelevant stimulus changes on stimulus and response repetition effects. Acta Psychologica, 112(2), 143-156.

Notebaert, W., Soetens, E., \& Melis, A. (2001). Sequential analysis of a Simon task - evidence for an attention shift account. Psychological Research, 65, 170-184.

Pashler, H., \& Baylis, G. C. (1991). Procedural learning: II. Intertrial repetition effects in speeded-choice tasks. Journal of Experimental Psychology: Learning, Memory, and Cognition, 17(1), 33-48.

Posner, M. I. (1980). Orienting of attention. Quarterly Journal of Experimental Psychology, 32(1), 3-25.

Posner, M. I., \& Cohen, Y. (1984). Components of visual orienting. Attention and Performance X: Control of Language Processes, 32, 531-556.

Posner, M. I., Rafal, R. D., Choate, L. S., \& Vaughan, J. (1985). Inhibition of return: Neural basis and function. Cognitive Neuropsychology, 2(3), 211-228.

Pratt, J., \& Castel, A. D. (2001). Responding to feature or location: A reexamination of inhibition of return and facilitation of return. Vision Research, 41(28), 3903-3908.

Prime, D. J., Visser, T. A., \& Ward, L. M. (2006). Re-orienting attention and inhibition of return. Perception \& Psychophysics, 68(8), 1310 1323.
Rabbitt, P. M. (1968). Repetition effects and signal classification strategies in serial choice-response tasks. The Quarterly Journal of Experimental Psychology, 20(3), 232-240.

Rabbitt, P. M. A., \& Rogers, M. (1965). Age and choice between responses in a self-paced repetitive task. Ergonomics, 8(4), 435-444.

Rajsic, J., Bi, Y., \& Wilson, D. E. (2014). Long-term facilitation of return: A response-retrieval effect. Psychonomic Bulletin \& Review, 21(2), 418-424.

Samuel, A. G., \& Kat, D. (2003). Inhibition of return: A graphical metaanalysis of its time course and an empirical test of its temporal and spatial properties. Psychonomic Bulletin \& Review, 10(4), 897-906.

Schvaneveldt, R. W., \& Chase, W. G. (1969). Sequential effects in choice reaction time. Journal of Experimental Psychology, 80(1), 1-8.

Smith, D. T., \& Schenk, T. (2012). The premotor theory of attention: Time to move on? Neuropsychologia, 50(6), 1104-1114.

Soetens, E. (1998). Localizing sequential effects in serial choice reaction time with the information reduction procedure. Journal of Experimental Psychology: Human Perception and Performance, 24(2), 547.

Soetens, E., Boer, L. C., \& Hueting, J. E. (1985). Expectancy or automatic facilitation? Separating sequential effects in two-choice reaction time. Journal of Experimental Psychology: Human Perception and Performance, 11(5), 598-616.

Spadaro, A., He, C., \& Milliken, B. (2012). Response to an intervening event reverses nonspatial repetition effects in $2 \mathrm{AFC}$ tasks: Nonspatial IOR? Attention, Perception, \& Psychophysics, 74(2), 331-349.

Spadaro, A., \& Milliken, B. (2013). Subjective Expectancy and Inhibition of Return: A Dissociation in a Non-Spatial Two-Alternative Forced Choice Task. Psicologica: International Journal of Methodology and Experimental Psychology, 34(2), 199-219.

Tanaka, Y., \& Shimojo, S. (1996). Location vs feature: Reaction time reveals dissociation between two visual functions. Vision Research, 36(14), 2125-2140.

Taylor, T. L., \& Donnelly, M. P. (2002). Inhibition of return for target discriminations: The effect of repeating discriminated and irrelevant stimulus dimensions. Perception \& Psychophysics, 64(2), 292-317.

Taylor, T. L., \& Ivanoff, J. (2005). Inhibition of return and repetition priming effects in localization and discrimination tasks. Canadian Journal of Experimental Psychology/Revue Canadienne de Psychologie Expérimentale, 59(2), 75-89.

Taylor, T. L., \& Klein, R. M. (2000). Visual and motor effects in inhibition of return. Journal of Experimental Psychology: Human Perception and Performance, 26(5), 1639-1656.

Terry, K. M., Valdes, L. A., \& Neill, W. T. (1994). Does "inhibition of return" occur in discrimination tasks? Perception \& Psychophysics, 55(3), 279-286.

Tipper, S. P., Weaver, B., \& Milliken, B. (1995). Spatial negative priming without mismatching: Comment on Park and Kanwisher (1994).

Tretyak, V., \& Yarrow, K. (2014). Motor plans persist to influence subsequent actions with four or more response alternatives. Acta Psychologica, 149, 9-17. 